\title{
Floating teeth in systemic sclerosis
}

\author{
Jae-Bum Jun ${ }^{1}$, Seunghun Lee ${ }^{2}$, and Jiyoon Bae $^{3}$
}

Departments of ${ }^{1}$ Rheumatology and ${ }^{2}$ Radiology, Hanyang University Hospital for Rheumatic Diseases, Seoul; ${ }^{3}$ Department of Pathology, National Police Hospital, Seoul, Korea

Received: March 5, 2020

Revised : March 24, 2020

Accepted: March 24, 2020

\section{Correspondence to}

Seunghun Lee, M.D.

Tel: +82-2-2290-9164

Fax: +82-2-2293-2111

E-mail: radsh@hanyang.ac.kr https://orcid.org/0000-00024348-7993
A 42-year-old woman presented to the Department of Dentistry with a 1-year history of loosening of multiple teeth in the mandible. Her history included difficulty in chewing food because of mobile teeth, and reduced mouth opening. She was diagnosed with systemic sclerosis 10 years ago. Intra-oral examination showed tongue rigidity and limited tongue movement. There was generalized gingival inflammation. Panoramic radiography of the jaw showed bone resorption in the right mandible and an exaggerated radiolucent area between the loosening teeth and the mandible corresponding to the location of a thickened periodontal membrane (Fig. 1A, arrows). Additionally, bone resorption of the mandibular body and angles were noted on both sides of the mandible (Fig. 1A). After one month, extractions of multiple loosening teeth were performed in the mandible. In a follow-up panoramic view taken a year later, newly developed loosening of the left maxillary molar tooth was observed (Fig. 1B, arrow). Extra-oral and oral examination showed taut, thickened, mask-like fascial skin, xerostomia, and a small oral orifice (Fig. 2).

Systemic sclerosis is a disorder of the connective tissue, characterized by fibrosis of the skin, blood vessels, visceral organs, and mucosa. Women are affected three to four times more frequently than men. Oral and facial tissues are often affected. The most common dental manifestations are the widening of the periodontal space and the loosening of the teeth, which occur in the absence of any significant periodontal disease. The loosening of the teeth is caused by the excess deposition of collagen fibers and collagen sclerosis, coupled with a decrease in the
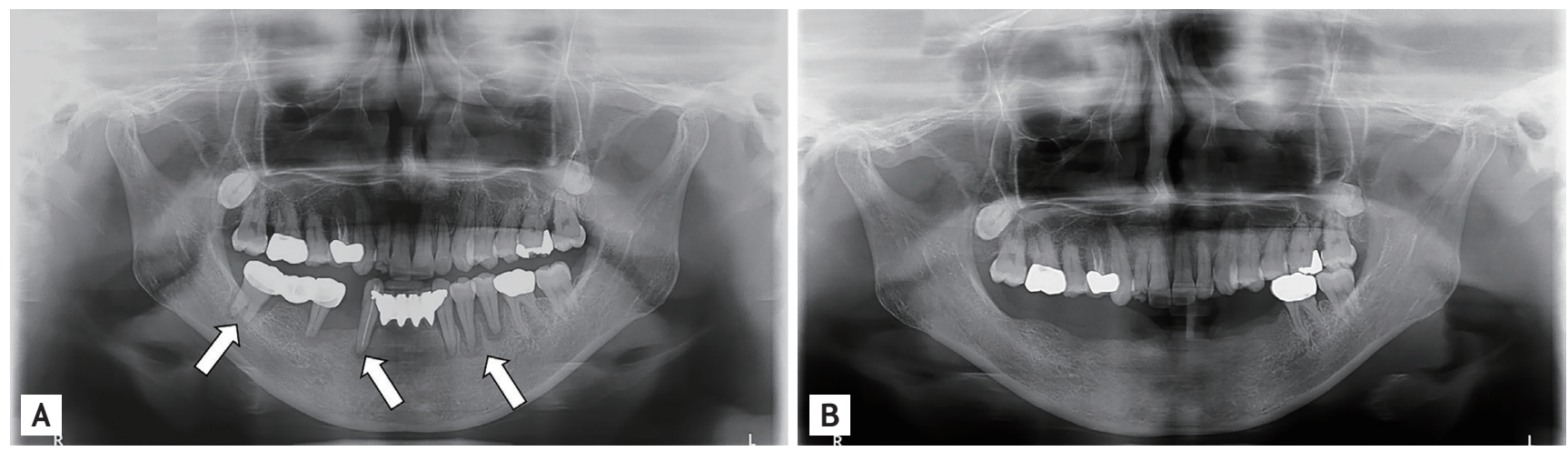

Figure 1. (A) The panoramic view of the jaw showed a widening of the periodontal radiolucency in the mandibular teeth (arrows). (B) After 1 year, a follow-up panoramic view of the jaw was obtained. Most of the mandibular teeth were not visible. Newly developed periodontal radiolucency was noted in the left maxillary molar tooth (arrow). 


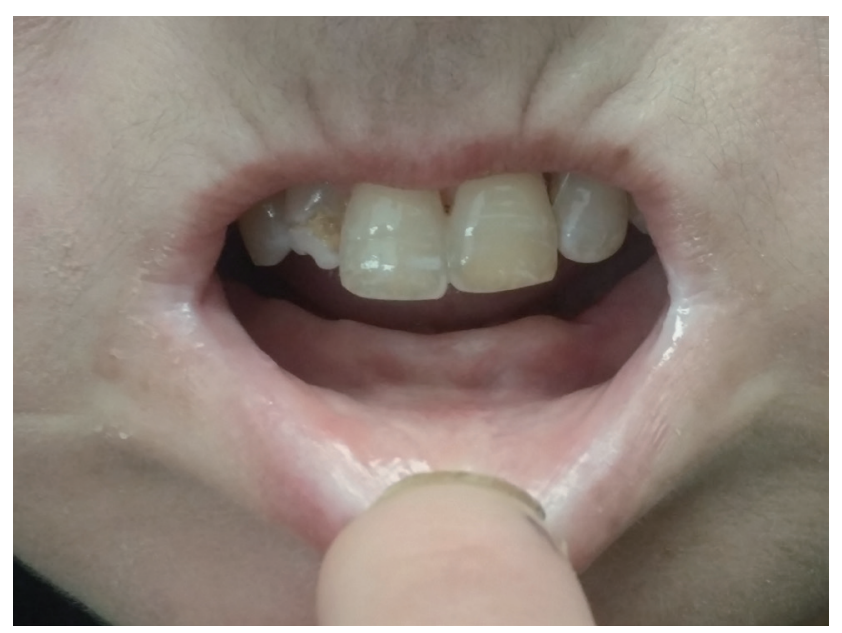

Figure 2. After 1 year, limited mouth opening and pronounced labial wrinkles were shown. Most of the mandibular teeth were not visible. number of connective tissue cells. Based on this case, we suggest that early monitoring of oral changes in patients with systemic sclerosis is mandatory and may improve living conditions.

Institutional Review Board approval was waived by the IRB committee (Hanyang University Hospital, 2018-07037) because this study did not collect or record sensitive personal information. All authors declare that written informed consent was obtained from the patient for publication of this case report and accompanying images.

\section{Conflict of interest}

No potential conflict of interest relevant to this article was reported. 